\title{
On optimal Hybrid ARQ control schemes for HSDPA with 16QAM
}

\author{
Simon Bliudze \\ LIX, École Polytechnique \\ 91128 Palaiseau, France \\ Email: bliudze@lix.polytechnique.fr
}

\author{
Nicolas Billy \\ Alcatel CIT, 1 rue Nieuport \\ 78148 Vélizy-Villacoublay, France \\ Email: nicolas.billy@alcatel.fr
}

\author{
Daniel Krob \\ LIX, École Polytechnique \\ 91128 Palaiseau, France \\ Email: dk@lix.polytechnique.fr
}

\begin{abstract}
We consider several Hybrid ARQ (H-ARQ) control schemes for High Speed Downlink Packet Access (HSDPA) with 16 symbols Quadrature Amplitude Modulation (16QAM). These schemes consist of a sequence of values for the $X_{r v}$ parameter to be used at sequential retransmissions when a block is not decoded correctly. A choice of an H-ARQ control scheme influences two parameters: Quality of Service (QoS) and User Equipement (UE) buffer requirements. Based on several link level simulations, we propose an optimal control scheme using maximum space, as well as two slightly suboptimal ones that allow to reduce the the UE buffer size.
\end{abstract}

Keywords - 16QAM constellation rearrangement, HSDPA, Hybrid ARQ, UMTS

\section{INTRODUCTION}

One of the latest features of the Universal Mobile Telecommunications System (UMTS) is the High Speed Downlink Packet Access (HSDPA) - a service allowing packet access at a very high bit-rate compared to previous releases. It is based on a new channel - High Speed Downlink Shared CHannel (HS-DSCH). Shared between several users, this channel is dedicated to downlink traffic and supports high data rates.

HS-DSCH is the main evolution of Release 5 (R5) of $3^{\text {rd }}$ Generation Partnership Project (3GPP) specifications. Release 5 introduces for this channel some new advanced radio technologies both in the physical and in the Medium Access Level (MAC) layer. The main techniques are: a new modulation scheme - 16-state Quadrature Amplitude Modulation (16QAM), adaptive modulation and coding (AMC), and Hybrid Automatic Repeat reQuest (H-ARQ) - an improved method of retransmission of false blocks. These new technologies allow to achieve data rates of up to $30 \mathrm{Mbps}$.

In downlink, H-ARQ allows a User Equipement (UE) to automatically request a retransmission of a block it didn't manage to decode correctly. In previous releases of UMTS (R99), with H-ARQ type I, on reception of a false block the UE discarded it and waited for a retransmission of the block from the Radio Network Controller (RNC) hoping to decode the new copy. In HSDPA fast H-ARQ is applied by retransmitting directly from Node B in the physical layer, thus enabling quicker retransmissions.

In R5 H-ARQ Type II/III is added, whose aim is to enable combining a retransmission with previous transmissions to

Manuscript received December 17, 2004. increase the chances of correct decoding. The disadvantage of H-ARQ is that the UE needs to store the false blocks and add the new soft bits received to the previous ones it couldn't decode correctly, which requires additional memory and processing.

Although some research has been done to determine the optimal parameters for ARQ as such (see for example [1]), none is so far available in the context of H-ARQ for HSDPA as defined by $3 \mathrm{GPP}$ specifications.

In this paper we shall attempt to determine an optimal $\mathrm{H}$ ARQ control scheme in terms of quality of service and UE buffer requirements. In Section II we give an overview of $\mathrm{H}-$ ARQ as well as of the different techniques it involves, then in Section III we present several link level simulations we have performed, and finally we conclude in Section IV by indentifying an optimal control scheme in terms of quality of service, as well as two suboptimal ones showing slightly worse performance but allowing to reduce the size of the UE buffer.

\section{HYBRID ARQ}

H-ARQ is described in [2] and consists of the following three techniques:

- Chase combining $(C C)$ - if the received block doesn't have the correct Circular Redundancy Check (CRC) sequence, it is retransmitted and new values of soft bits are added to those of the first transmission.

- Incremental redundancy (IR) - incorrect block is retransmitted with different redundancy version parameters (different systematic over parity bits priority and/or rate matching parameters).

- 16QAM constellation rearrangement ( CoRe) - different mapping of blocks of bits to symbols.

Chase combining was originally proposed in [3]. It provides a considerable gain in transmission power $(3 \mathrm{~dB}$ in Gaussian environment) at the cost of slightly increased processing complexity and a buffer in the UE that is required to store the received values. Chase combining can be used at both bit and symbol levels. However, the improvement of performance is not sufficient to obtain target rates.

Incremental redundancy provides yet another improvement by allowing to send additional information in case were retransmission is needed. In other words, bits which are punctured at the rate matching step of the first transmission 
TABLE I

ENCODING OF REDUNDANCY VERSION PARAMETERS FOR 16QAM

\begin{tabular}{l|llllllll}
\hline$X_{r v}$ & 0 & 1 & 2 & 3 & 4 & 5 & 6 & 7 \\
\hline$s$ & 1 & 0 & 1 & 0 & 1 & 1 & 1 & 1 \\
$r$ & 0 & 0 & 1 & 1 & 0 & 0 & 0 & 1 \\
$b$ & 0 & 0 & 1 & 1 & 1 & 2 & 3 & 0 \\
\hline
\end{tabular}

can be sent at the second one. More precisely, one can prioritise sending systematic or parity bits, and at the same time vary rate matching parameters, thus choosing not to puncture the same bits as at previous transmissions ${ }^{1}$. This technique greatly improves Turbo decoder's performance (see [4], [5] for a comparison of IR vs CC). The disadvantage is that the buffer size in the UE has to increase considerably, as well as processing complexity.

16QAM constellation rearrangement is a technique proposed in [6], [7] that allows to increase performance as compared to Chase combining while keeping processing complexity and buffer requirements comparatively low. Thus constellation rearrangement can be viewed as a low complexity alternative to incremental redundancy. As implied by its name, this technique is only applicable when 16QAM modulation is used $^{2}$, and consists in changing bits-to-symbols mapping. It is described in more detail in Section II-A.

Both incremental redundancy and 16QAM constellation rearrangement are controlled by a set of so-called redundancy version (RV) parameters: $r, s$, and $b$ that are in their turn determined by a single parameter $X_{r v}$. The parameters $r$ and $s$ control the rate matching step, which is the base of incremental redundancy technique, while $b$ controls the way 16QAM constellation is rearranged.

The value of $s$ can be either 0 or 1 and indicates if, at rate matching step, the systematic bits are prioritised $(s=1)$ or not $(s=0)$. Once we know what flows are to be punctured in priority - systematic or parity bits - the value of $r$ determines the exact puncturing pattern within these flows. The range of $r$ is 0 to 3 for the $\mathrm{QPSK}^{3}$ modulation or 0 to 1 for 16QAM. For 16QAM these parameters are encoded according to Table I (see also [2], p. 67).

The value of the $X_{r v}$ parameter is chosen in the following manner. We fix a list $X=\left\{x_{0}, x_{1}, \ldots, x_{l-1}\right\}$ of values between 0 and 7, where $l$ is arbitrary. We shall now set $X_{r v}=x_{n-1 \bmod l}$ at $n$-th transmission. In other words for each given block the value of $X_{r v}$ cycles through the list $X$ that we shall call the $H$-ARQ control scheme.

For example, a list consisting of a single value $\{0\}$ defines the scheme that only uses Chase combining (the same redundancy version is sent at each retransmission). A list

\footnotetext{
${ }^{1}$ If we choose to send the same redundancy version at each transmission, we obtain Chase combining. Thus the latter is a special case of Incremental redundancy.

${ }^{2}$ Here we place ourselves in the context of R5 UMTS where the only modulations in use are BPSK, QPSK, and 16QAM. In a different context constellation rearrangement could be used with any amplitude modulation (e.g. 64QAM, 128QAM, etc.)

${ }^{3}$ Quadrature Phase Shift Keying
}

with two elements $\{0,1\}$ implies that we send alternatively systematic and parity bits. If in the latter case a third or fourth retransmission is required the value of $X_{r v}$ shall again be 0 and 1 correspondingly.

The question arises naturally: what H-ARQ control scheme is optimal in terms of the quality of service (QoS) (lowest $I_{o r} / I_{o c}{ }^{4}$ for a given $\mathrm{BLER}^{5}$ ) and complexity (UE buffer and processing)? Before answering this question we shall analyse in more detail the 16QAM constellation rearrangement.

\section{A. 16QAM constellation rearrangement}

16QAM is a quadrature amplitude modulation based on a constellation of 16 symbols depicted in Fig. 1. The bits to be transmitted are grouped in blocks of four. Each one of these blocks defines a constellation symbol that is then transmitted over a communication channel. More precisely, denoting the four bits by $i_{1} q_{1} i_{2} q_{2}$ correspondingly, the complex-valued symbol is obtained with the following formula:

$$
s=\frac{\widetilde{\imath_{1}}\left(2-\widetilde{\imath_{2}}\right)+j \cdot \widetilde{q_{1}}\left(2-\widetilde{q_{2}}\right)}{\sqrt{5}},
$$

where $j=\sqrt{-1}$, and $\widetilde{b}=(-1)^{b}$ is the real-valued bit corresponding to the logical bit $b$. One can observe that first and third bits $\left(i_{1}\right.$ and $i_{2}$ ) define the real part of the symbol, and second and fourth $\left(q_{1}\right.$ and $\left.q_{2}\right)$ - the imaginary one, and therefore demodulation of the received signal $\widehat{s}$ would consist, firstly, in comparing its real and imaginary parts to zero in order to determine $i_{1}$ and $q_{1}$, and, secondly, in comparing absolute values of its real and imaginary parts to the threshold $2 C / \sqrt{5}$ to determine $i_{2}$ and $q_{2}$, where $C$ depends on the radio conditions and transmit power.

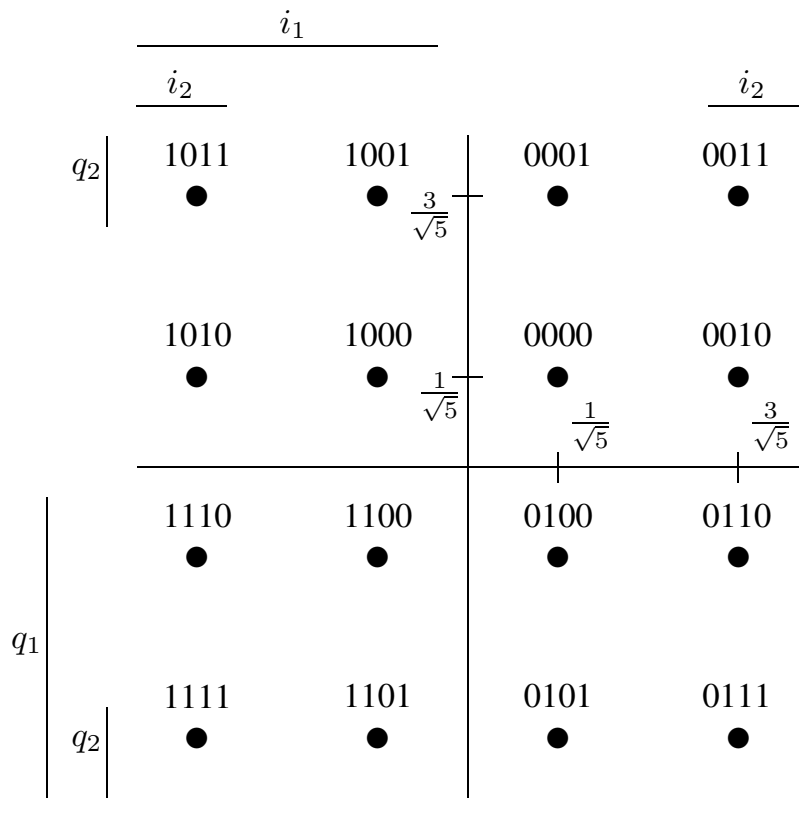

Fig. 1. 16QAM symbol constellation

\footnotetext{
${ }^{4}$ The ratio of the total user power to noise $(\mathrm{dB})$

${ }^{5}$ BLock Error Rate
} 
TABLE II

CONSTELLATION REARRANGEMENT FOR 16QAM (SEE [2], P. 65)

\begin{tabular}{c|c|l}
\hline $\begin{array}{c}\text { Constellation ver- } \\
\text { sion parameter } b\end{array}$ & $\begin{array}{c}\text { Output bit } \\
\text { sequence }\end{array}$ & Operation \\
\hline 0 & $i_{1} q_{1} i_{2} q_{2}$ & None (mapping as in Fig. 1) \\
1 & $i_{2} q_{2} i_{1} q_{1}$ & Swapping MSBs with LSBs \\
2 & $i_{1} q_{1} \overline{i_{2} q_{2}}$ & Inversion of LSBs' logical values \\
3 & $i_{2} q_{2} \overline{i_{1} q_{1}}$ & Both swapping and inversion \\
\hline
\end{tabular}

The advantage of 16QAM is that 4 bits are transmitted per single complex-valued symbol, as opposed to 2 in QPSK the modulation used for all channels in UMTS transmitting user data, except for HS-DSCH, - thus doubling the possible bit-rate. On the other hand, its disadvantage is a more complex modulation and demodulation procedure, as well as increased sensitivity to radio conditions.

It is well known (see for example [8]) that among the four bits forming a symbol in 16QAM the probability of error can be considerably less for the most significant bits (MSBs) than for the less significant bits (LSBs). For example, if we consider symbol 2 (0010) of the constellation in Fig. 1, for the first bit to be demodulated erroneously the perturbation of the real part of the transmited signal has to be three times that necessary to induce an error in the third bit.

In order to compensate for this effect, bits can be rearranged before retransmission in such a manner that some less protected bits become more protected. More precisely, denoting the four bits by $i_{1} q_{1} i_{2} q_{2}$, one of the four transformations in Table II is applied before they are mapped to a constellation symbol (see [2]).

According to the ergodic principle, averaging of error probability over a long chain of bits is equivalent to averaging it over the symbol constellation. Thus, in order to better understand the principle of constellation rearrangement, we can consider a transmission where each constellation symbol is sent exactly once over an ideal channel (no fading, no noise). Suppose we use standard bits-to-symbols mapping, i.e. $b=0$. We then have a chain of 64 bits, out of which 16 are better protected than the other 48 . These 16 bits are the two MSBs of each of four symbols in the corners of the constellation, and one of the MSBs for the eight other symbols on the constellation's exterior. Each of the four transformations in Table II provides better protection for a different set of 16 bits. Thus consequent retransmissions with different constellation arrangements would considerably improve Turbo decoder's performance.

One can make the following observations regarding constellation rearrangement.

- Constellation rearrangement does not require additional buffer in the UE. The only space required is that, necessary to store three additional tables for bits-tosymbols mapping, and it is negligible compared to the size of buffer used to store transmitted bits. There is no additional processing to be done.

- When only one transmission is performed, all four con- stellation rearrangement techniques are equivalent. Similarly, if several retransmissions are needed, whatever is the rearrangement sequence, there is always an equivalent one with first transmission using standard mapping $(b=$ $0)$. Maximum benefit from constellation rearrangement can be obtained with four retransmissions using different rearrangement techniques.

\section{B. Candidate $H-A R Q$ schemes}

As it has been mentioned above, the maximum benefit from constellation rearrangement can be obtained with four retransmissions. The same can be said about incremental redundancy in 16QAM as there are four different redundancy versions: systematic or parity bits, and two rate matching patterns for each of them. We shall therefore compare different H-ARQ control schemes of length 4 . The schemes we compare are shown in Table III.

Along with the list defining a scheme and its short description we give in this table the scheme's space complexity index. This index represents the number of redundancy versions (systematic/priority bits, rate matching pattern) used in the scheme. Each additional redundancy version increments the number of bits that have to be stored in the UE buffer. Therefore the higher is the index in question, the more expensive the scheme is in terms of buffer requirements.

Let us briefly discuss the proposed schemes.

CC - as indicated in Table III this scheme represents Chase combining. Indeed, the same bits are sent at all retransmissions.

CoRe - this scheme makes full use of constellation rearrangement, but none of incremental redundancy: we send the same bits at each transmission using all possible bits-to-symbols mappings.

IR - in this scheme we send alternatively systematic and parity bits. Two last retransmissions use a different rate matching pattern compared to the first two, thus making full use of incremental redundancy.

IR+ - incremental redundancy is enhanced here by using constellation rearrangement on the last two retransmissions. This increases protection of bits that are not punctured in both rate matching patterns.

IR/2-a - in this scheme only systematic bits are sent. Thus, comparing its performance with that of other schemes we can see if alternating systematic bits with parity ones is preferable to alternating rate matching patterns.

IR/2-b - this scheme is complementary to IR/2-a: we send systematic and parity bits alternatively maintaining the same rate matching pattern.

CoReIR- - this scheme is an attempt at a compromise between the high performance and high complexity of IR on one hand, and lower performance and low complexity of CoRe on the other: we alternate systematic and parity bits, but instead of using different rate matching patterns, we use different bits-to-symbols mapping. As there is no available value of $X_{r v}$ that 
TABLE III

COMPARED H-ARQ CONTROL SCHEMES.

\begin{tabular}{|l|c|c|l|}
\hline Name & Scheme & UE buffer & Description \\
\hline CC & $\{0\}$ & 1 & Chase combining \\
CoRe & $\{0,4,5,6\}$ & 1 & Constellation rearrangement \\
IR & $\{0,1,7,3\}$ & 4 & Incremental redundancy \\
IR+ & $\{0,1,2,3\}$ & 4 & Incremental redundancy with constellation rearrangement \\
IR/2-a & $\{0,7\}$ & 2 & Systematic bits only with two rate matching patterns \\
IR/2-b & $\{0,1\}$ & 2 & Systematic then parity bits; only one rate matching pattern \\
CoReIR- & $\{0,1,4,1\}$ & 2 & Hybrid sub-optimal \\
CoReIR & $\{0,1,4,8\}$ & 2 & Hybrid (CoRe + IR) \\
\hline
\end{tabular}

A scheme here is a list of values for $X_{r v}$ to be used at corresponding retransmissions (cf. Table I).

would have $s=0, r=0$, and $b \neq 0$ (as $X_{r v}=1$, but with a different bits-to-symbols mapping), we use the same value of $X_{r v}$ for second and fourth transmissions.

CoReIR - same as CoReIR-, but we introduce for testing purposes $X_{r v}=8$ that is not in the 3 GPP specifications (Table I; also [2]) and encodes the following combination of RV parameters: $s=0, r=0, b=1$, i.e. prioritising parity bits with the same rate matching pattern as for $X_{r v}=1$ but with a different bits-tosymbols mapping.

The roles of these schemes are as follows. CC provides us with reference performances. IR+ being the scheme that ensures most diversity (different redundancy versions plus some use of constellation rearrangement), is the candidate for best performance, however it also requires the largest UE buffer. The goal of CoRe and IR is to enable a comparison between the two techniques, as well as to verify if sufficiently good results can be obtained using only one of them (initially constellation rearrangement was proposed in [6] to completely replace incremental redundancy). Furthermore, IR/2-a and IR/2-b allow us to single out the aspect of incremental redundancy that ensures the most gain in performance: IR/2-a does not send parity bits only varying the rate matching pattern, whereas IR/2-b does the inverse by alternating systematic and parity bits. Finally, as it has been mentioned above, CoReIR and CoReIR- are constructed by merging together incremental redundancy and constellation rearrangement in order to obtain good performances while keeping low UE buffer requirements.

\section{Simulations}

\section{A. Simulation conditions}

For all of the above H-ARQ schemes we perform link level simulations in Gaussian environment. We consider an HSDPA connection using 16QAM with one channelisation code at coding rate $3 / 4$, and we consider BLER to $I_{o r} / I_{o c}$ ratio at different retransmissions. We implement the full HSDPA processing chain, of which the coding part is shown in Fig. 2. The list of simulation parameters is given in Table IV.

\section{B. Results}

Fig. 3 shows BLER performance of all considered H-ARQ control schemes after second transmission. In solid lines are plotted the curves for schemes that do not send parity bits on the second transmission, while those that do are plotted in dashed lines ${ }^{6}$.

One can observe in the first place that sending parity bits provides a gain of approximately $1.8 \mathrm{~dB}$ at $10 \%$ BLER over resending systematic ones. Another observation to be made is that sending systematic bits with the same rate matching pattern but a different bits-to-symbols mapping (CoRe) provides a gain of approximately $0.4 \mathrm{~dB}$ over using a different rate matching pattern with the same mapping (IR/2-a).

Comparing performances after the third transmission (see Fig. $4^{6}$ ) allows us to differentiate between the schemes that produced similar results after second transmission. As expected, IR+ provides best performance vis-à-vis the BLER to $I_{o r} / I_{o c}$ ratio.

Both CoReIR and CoReIR- perform approximately $0.4 \mathrm{~dB}$ better than IR (cf. the second observation on the performances after two transmissions). The difference between IR+ and CoReIR (CoReIR-) is very slight (less than $0.1 \mathrm{~dB}$ ). Thus, up to this point both CoReIR and CoReIR- provide performances close to optimal, while maintaning lower UE buffer requirements (see again Table III).

Let us finally compare the performances after the fourth transmission (Fig. 5). We can observe that due to retransmitting the same redundancy version as at the second transmission CoReIR- performs here worse than IR. At the same time CoReIR, which has a much lower requirements for UE buffer, provides a considerably better performance. Indeed, its performance is approximately $0.2 \mathrm{~dB}$ better than that of IR, and very close to IR+.

To finalise the presentation of simulation results we give in Table V the ratings of all schemes considered, and in Fig. 6 the bit-rate achieved by the best candidates compared to that of Chase combining.

\section{CONCLUSION}

In this paper we have studied several H-ARQ control schemes. These schemes were chosen in such a way as to isolate either different H-ARQ techniques (Chase combining, incremental redundancy, and constellation rearrangement), or their more specific aspects (sending systematic or parity bits in priority, or using different rate matching patterns). Our goal

\footnotetext{
${ }^{6}$ Note that after second and third retransmissions performances are the same for some schemes, therefore all dashed curves in Fig. 3 and Fig. 4 coincide.
} 


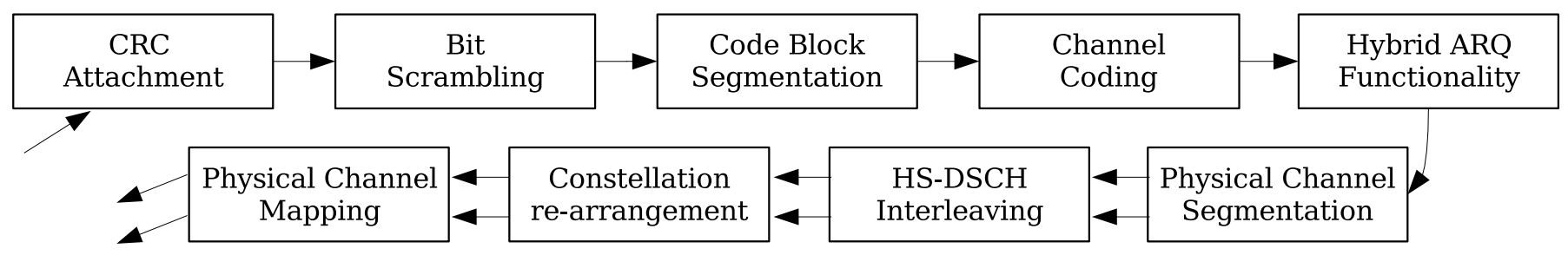

Fig. 2. HSDPA coding chain

TABLE IV

LIST OF SIMULATION PARAMETERS

\begin{tabular}{|l|l|}
\hline Parameter & Value \\
\hline \hline Channel model & Additive White Gaussian Noise (AWGN) \\
Chip-rate & $3.84 \mathrm{Mcps}$ \\
Power control & Off \\
Channel estimation & Ideal \\
Allocated power for HS-DSCH & $80 \%(-1 \mathrm{~dB})$ \\
\hline Spreading factor & 16 \\
Number of codes for HS-DSCH & 1 \\
Number of slots per TTI & 3 \\
Frame length & $2 \mathrm{~ms}$ \\
Number of transport blocks per TTI & 1 \\
\hline Channel coding & Turbo code (rate 3/4) \\
CRC & 24 bits \\
Tail bits & 12 \\
Turbo decoder & Log-MAP \\
Number of decoder iterations & 8 \\
Max number of retransmissions & 4 \\
\hline Modulation & $16 \mathrm{QAM}$ \\
Accuracy & $50000-250000$ slots per $I_{o r} / I_{o c}$ value; At least 100 \\
\hline
\end{tabular}

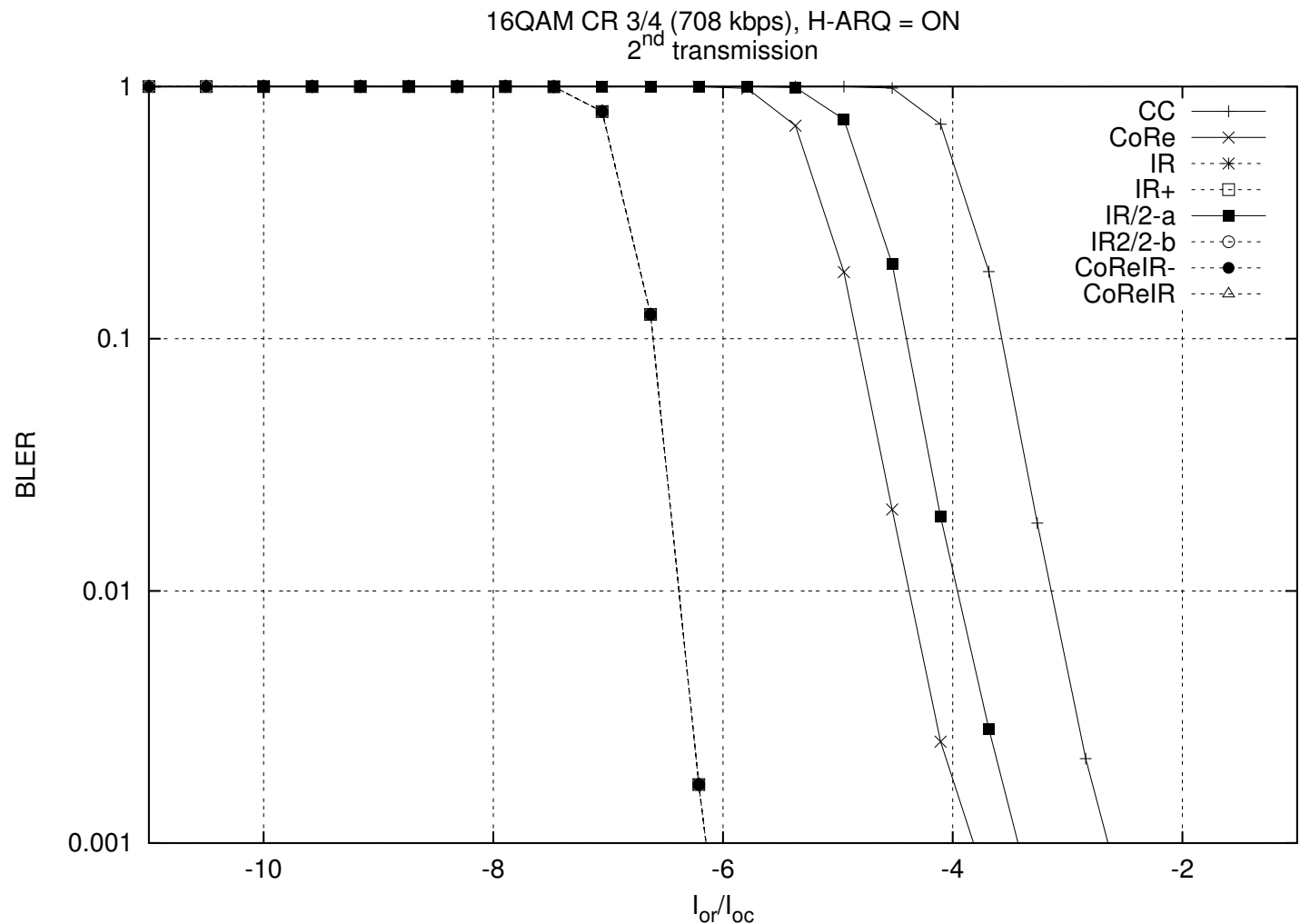

Fig. 3. Performance of different H-ARQ control schemes after $2^{\text {nd }}$ transmission 


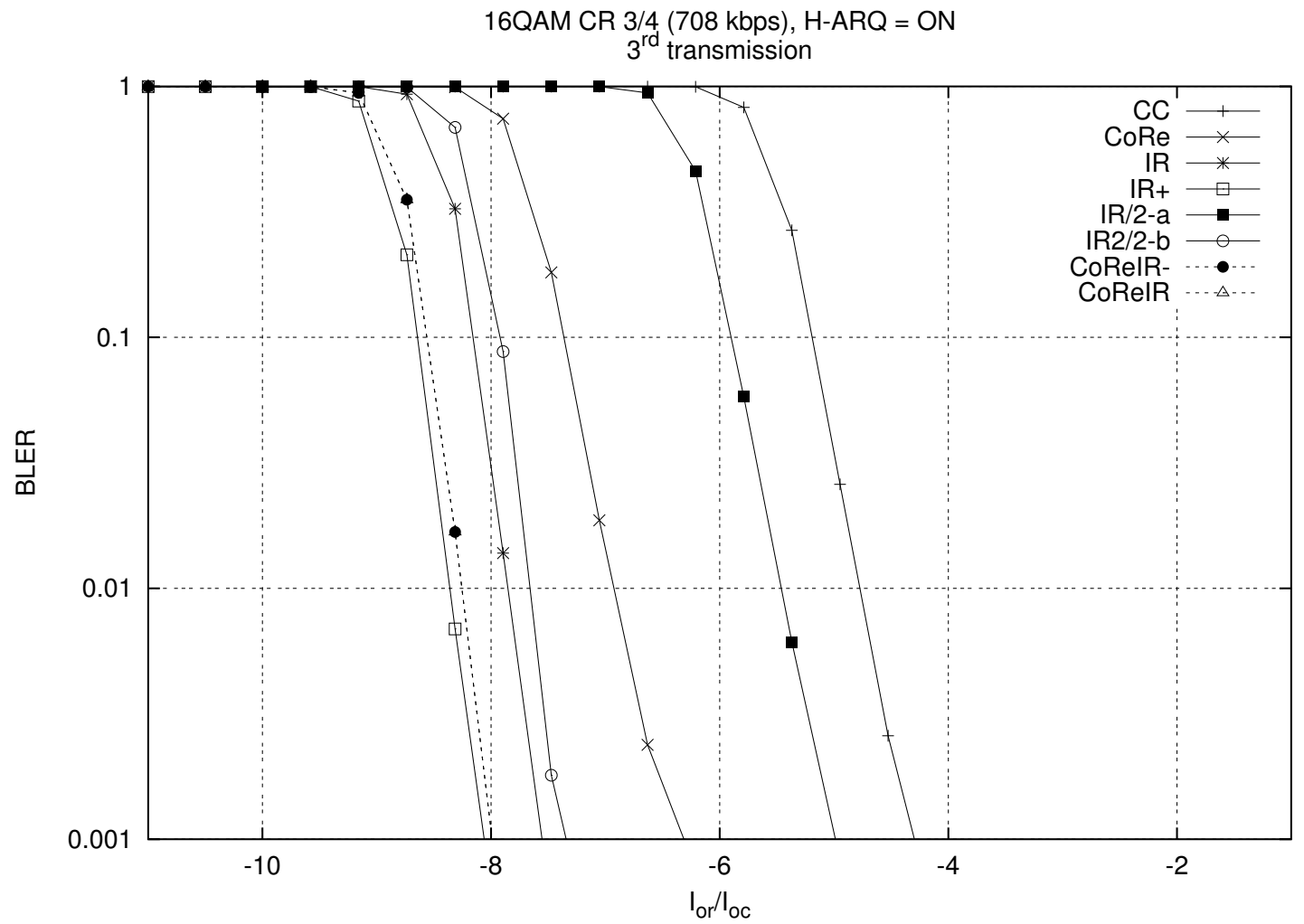

Fig. 4. Performance of different H-ARQ control schemes after $3^{\text {rd }}$ transmission

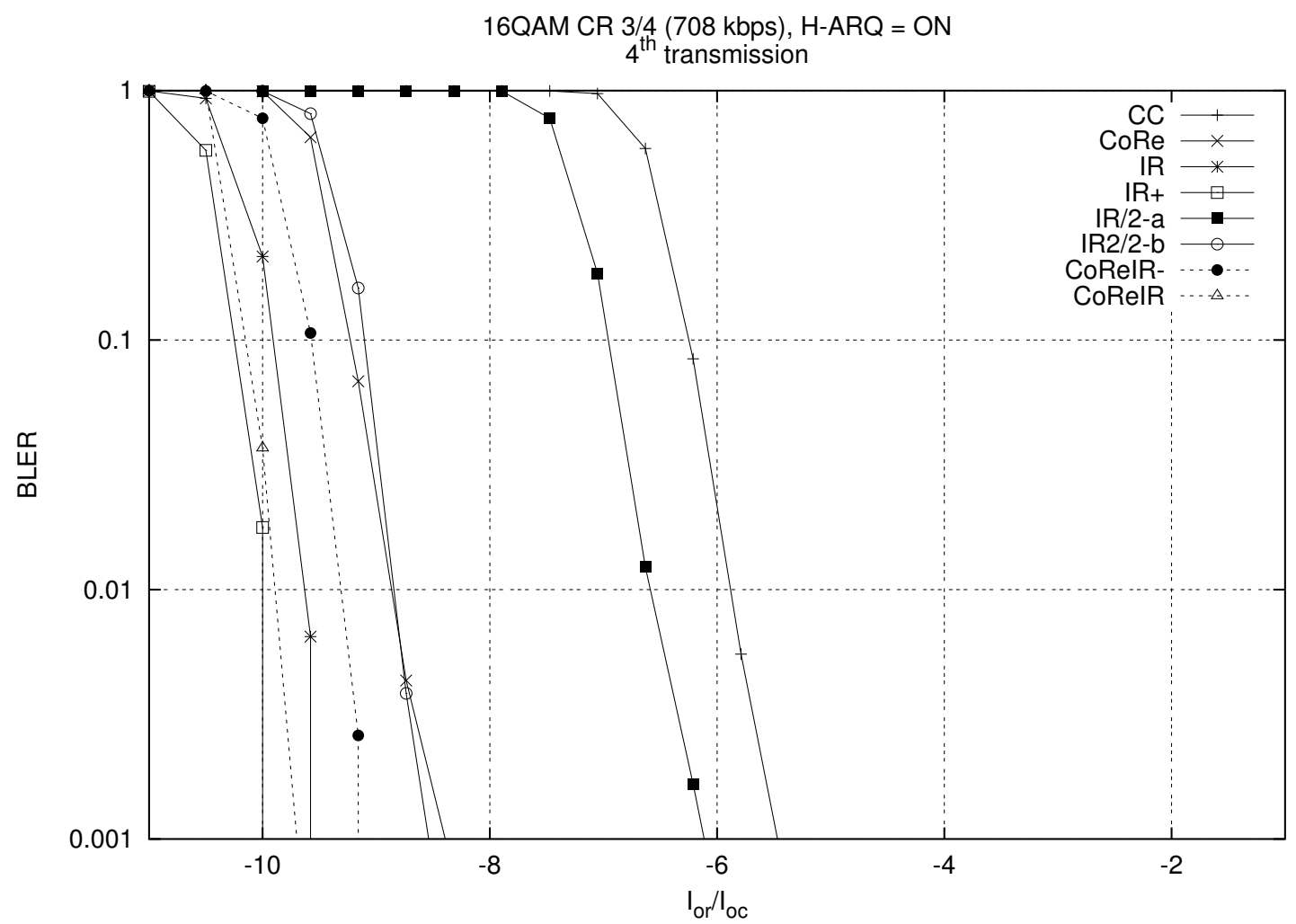

Fig. 5. Performance of different H-ARQ control schemes after $4^{\text {th }}$ transmission 


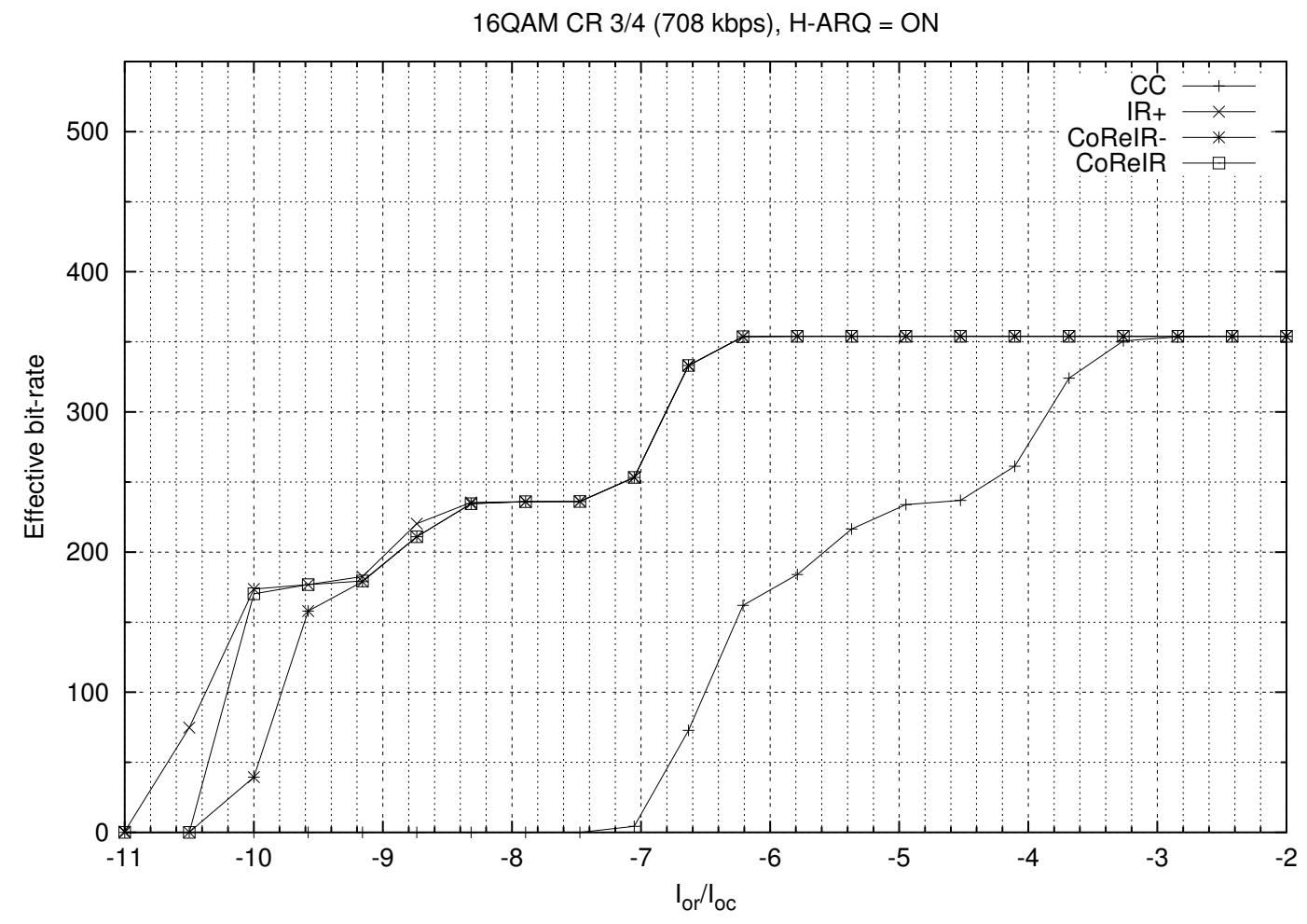

Fig. 6. Effective bit-rate achieved with best H-ARQ control schemes

TABLE V

BLER TO $I_{o r} / I_{O C}$ RANKING OF ALL SCHEMES AFTER EACH RETRANSMISSION

\begin{tabular}{|l|c|c|c|c|}
\hline Name & UE buffer & $2^{\text {nd }}$ & $3^{\text {rd }}$ & $4^{\text {th }}$ \\
\hline CC & 1 & 8 & 8 & 8 \\
CoRe & 1 & 6 & 6 & 5 \\
IR & 4 & $1-5$ & 4 & 3 \\
IR+ & 4 & $1-5$ & 1 & 1 \\
IR/2-a & 2 & 7 & 7 & 7 \\
IR/2-b & 2 & $1-5$ & 5 & 6 \\
CoReIR- & 2 & $1-5$ & $2-3$ & 4 \\
CoReIR & 2 & $1-5$ & $2-3$ & 2 \\
\hline
\end{tabular}

was to determine the optimal scheme in terms of BLER to $I_{o r} / I_{o c}$ performance and UE buffer requirements.

The best performance in terms of BLER to $I_{o r} / I_{o c}$ ratio is provided by the scheme we have denoted IR+ that makes full use of both incremental redundancy and constellation rearrangement. However - and for that reason, - this scheme requires the biggest UE buffer among all possible schemes.

At the same time another scheme denoted CoReIR provides performance that is only about $0.1 \mathrm{~dB}$ worse than that of IR+ while considerably reducing UE buffer requirements ${ }^{7}$.

The disadvantage of this scheme is that at the fourth transmission it uses a combination of redundancy version parameters that is not found in the 3GPP specifications, i.e. $s=0, r=0$, and $b=1$ (transmitting parity bits with the same

\footnotetext{
${ }^{7} \mathrm{UE}$ supporting all coding rates from $1 / 3$ to 1 would require approximately $50 \%$ more buffer with IR+ than with CoReIR.
}

rate matching pattern as for $X_{r v}=1$, but with a different bitsto-symbols mapping).

Therefore there are two possible lines of action:

1) Without changing the specifications (see Table I), one should decide between IR+ and CoReIR- according to what is being prioritised: performance or UE buffer size.

2) One of the entries of the Table I $\left(X_{r v}=5,6\right.$, or 7$)$ should be modified to match the parameters specified above, and CoReIR should be selected as optimal HARQ control scheme.

\section{REFERENCES}

[1] V. Mitlin, "Optimal selection of ARQ parameters in QAM channels," Wireless Communications and Mobile Computing, to appear, DOI $10.1002 / \mathrm{wcm} .206$.

[2] Multiplexing and channel coding (FDD), 3rd Generation Partnership Project (3GPP) Technical Specification TS 25.212, Rev. 5.9.0, June 2004.

[3] D. Chase, "Code combining: A maximum-likelihood decoding approach for combining an arbitrary number of noisy packets," IEEE Trans. Commun., vol. 33, pp. 593-607, May 1985.

[4] Performance Comparison of Hybrid-ARQ Schemes, 3rd Generation Partnership Project (3GPP) Technical Specification TSGR1\#17(00)1396, Oct. 2000.

[5] Performance Comparison of Chase Combining and Incremental Redundancy for HSDPA, 3rd Generation Partnership Project (3GPP) Technical Specification TSGR1\#17(00)1428, Nov. 2000.

[6] Enhanced HARQ Method with Signal Constellation Rearrangement, 3rd Generation Partnership Project (3GPP) Technical Specification TSGR1\#19(01)0237, Mar. 2001.

[7] Further Simulation Results on HARQ with Signal Constellation Rearrangement, 3rd Generation Partnership Project (3GPP) Technical Specification TSGR1\#20(01)0537, May 2001.

[8] J. Proakis, Digital Communications, 3rd ed. McGraw-Hill, 1995. 